\title{
Short Communication: \\ Floristic survey of vascular plant in the submontane forest of Mt. Burangrang Nature Reserve, West Java, Indonesia
}

\author{
TRI CAHYANTO ${ }^{1, \vartheta}$, MUHAMMAD EFENDI ${ }^{2, \vartheta \vartheta}$, RICKY MUSHOFFA SHOFARA ${ }^{1,}$ MUNA DZAKIYYAH ${ }^{1}$, \\ NURLAELA ${ }^{1}$, PRIMA G. SATRIA ${ }^{1}$ \\ ${ }^{1}$ Department of Biology, Faculty of Science and Technology,Universitas Islam Negeri Sunan Gunung Djati Bandung. Jl. A.H. Nasution No. 105, \\ Cibiru,Bandung 40614, West Java, Indonesia. Tel./fax.: +62-22-7800525, `email: tri_cahyanto@uinsgd.ac.id \\ ${ }^{2}$ Cibodas Botanic Gardens, Indonesian Institute of Sciences. J1. Kebun Raya Cibodas, Sindanglaya, Cipanas, Cianjur 43253, West Java, Indonesia. \\ Tel./fax.: +62-263-512233, ^vemail: muhammadefendi05@gmail.com
}

Manuscript received: 1 July 2019. Revision accepted: 18 July 2019.

\begin{abstract}
Cahyanto T, Efendi M, Shofara RM. 2019. Short Communication: Floristic survey of vascular plant in the submontane forest of Mt. Burangrang Nature Reserve, West Java, Indonesia. Biodiversitas 20: 2197-2205. A floristic survey was conducted in submontane forest of Block Pulus Mount Burangrang West Java. The objectives of the study were to inventory vascular plant and do quantitative measurements of floristic composition as well as their structure vegetation in the submontane forest of Nature Reserves Mt. Burangrang, Purwakarta West Java. Samples were recorded using exploration methods, in the hiking traill of Mt. Burangrang, from 946 to $1110 \mathrm{~m}$ asl. Vegetation analysis was done using sampling plots methods, with plot size of $500 \mathrm{~m}^{2}$ in four locations. Result was that 208 species of vascular plant consisting of basal family of angiosperm (1 species), magnoliids ( 21 species), monocots (33 species), eudicots (1 species), superrosids (1 species), rosids (74 species), superasterids (5 species), and asterids (47), added with 25 species of pterydophytes were found in the area. The three families of plants are Lauraceae (10 species), Urticaceae ( 9 species), and Rubiaceae (8 species) dominating those areas. Fourteen species belong to IUCN red list: Least concern/LC (12 species), Vulnerable/VU (1 species), and endangered/EN (1 species). Furthermore, Castanopsis argentea A.DC, Pinanga javana Blume and Amorphophallus decus-silvae Backer \& Aldrew belonging to protected plants are also found in the area. Based on the assessment of analysis vegetation, the forest has experienced disturbance, the density of trees is commonly low and has a lot of gaps. Many vacant lots are found. On the other side, there is the presence of invasive plant species that may slow down a succession into climax growth of local plant.
\end{abstract}

Keywords: Amorphophallus decus-silvae, ecology, Mt. Burangrang, plant conservation, vascular plant

\section{INTRODUCTION}

The mountain forests, one of them in Java Island area, are the last zone of in situ conservation of plant species from deforestation and exchanging of land which has been carried out in the last nearest time. The requirement of food and settlement has pressed to open much larger land (Setiawan and Sulistyawati 2008; van Welzen and Raes 2011; Tsujino et al. 2016). On the other hand, diversity loss and dynamic vegetation succession that were often found in the mountain forest of Java (Zuhri and Mutaqien 2011; Purwaningsih et al. 2017; Zuhri et al. 2018), will encourage innovation to the conservation of the flora on every mountain, such as in Burangrang Nature Reserve.

Mount Burangrang NR is part of the tropics mountain in West Java; this area is up to 2.700 ha and covers two regencies namely Purwakarta and Subang (BBKSDA 2016). Ecologically, Burangrang Nature Reserve area has an important part as a territory for water catching and water reservoirs for its surrounding area. Besides that, Mt. Burangrang becomes a natural habitat of Java primate, such as owa java, lutung and surili, so that flora conservation to support the animals woof must be regarded (BBKSDA 2016). On the other hand, some areas of Burangrang Nature Reserve are adjacent immediately with agriculture and plantation so the plants are susceptive to be coming into natural forest, as reported by Zuhri et al. (2018) in Mount Gede.

The book Flora of Java composed by Backer and Bakhuizen v.d. Brink $(1963 ; 1965 ; 1968)$ in comprehensive way consists of the description of flora in Java and The Mountain Flora of Java (van Steenis 1972; 2006). Specifically, this book describeshighlands flora and it becomes the most references inventory of the flora in Mount Burangrang Nature Reserve. More than 400 species of highlands flora, including Nephenthes gymnamphora, Morinda sarmentosa, and Vernonea cymosa are found in Mt. Burangrang Nature Reserve area (Van Steenis 2006). However, the list of flora must be made clear. So, this reseach is purposeful to get the data about the kinds of flora in the hills forest of Mount Burangrang Nature Reserve, Purwakarta, West Java, Indonesia.

\section{MATERIALS AND METHODS}

\section{Study area}

The study was conducted in the submontane forest on Mt. Burangrang Nature Reserve, Purwakarta District, West Java Province, Indonesia at 946 to $1104 \mathrm{~m}$ asl., in 
coordinates: $06^{\circ} 43^{\prime} 37.8^{\prime \prime} \mathrm{S}$ to $6^{\circ} 43^{\prime} 441^{\prime \prime} \mathrm{S}$ and $107^{\circ} 33^{\prime} 21.5^{\prime \prime} \mathrm{E}$ to $107^{\circ} 33^{\prime} 07.8^{\prime \prime}$ E. The topography condition of the study site was rather flat and uphill with slopes of $10-45^{\circ}$. Microclimate measurements were carried out during study, i.e. temperature $\left(23.2-27.9^{\circ} \mathrm{C}\right.$, in the afternoon), air humidity (up to $92 \%$ ), $\mathrm{pH}$ (6.2-7.2), soil humidity (50 to 90\%), and light intencity (137 to 2240 lux).

\section{Procedures}

There are two types of data taken from the site, i.e. (i) list of vascular plants, both in the plot and around observation plots, and (ii) data of floristic composition and vegetation structure.

\section{Inventory of vascular plants}

Sampel were recorded using exploration methods (Rugayah et al. 2004), in the hiking traill of Mt. Burangrang Purwakarta ( $\pm 3.2 \mathrm{~km}$ length) (Figure 1). The Species were recorded based on their scientific names and families, while the unidentified species were made herbarium voucher referring to de Vogel (1987), to be identified later using an identification book such as Flora of Java (Backer and Bakhuizen v.d. Brink 1963; 1965; 1968), Varenflora voor Java (Backer and Posthumus 1939), The Mountain Flora of Java (van Steenis 2006), A Revised Flora of Malaya (Holttum 1966), Flora Malesiana Vol. 4 (2012) and other papers (Zhu et al. 2012; Girmansyah 2008; Hadiah 2007). The naming of species, genera, families and taxon level refers to the Angiosperm Phylogeny Group classification (1998; 2003; 2009; 2016) for flowering plants, while for lycophytes and fern, it refers to Christenhusz et al. (2011) and Rothfels et al. (2012).

\section{Vegetation sampling}

Collecting data procedure was done by the vegetation analysis technique using purposive sampling (plot) in depended path. Sampling was done using four plots in altitude of $946-1110 \mathrm{~m}$ asl.. Every plot has magnitude of $10 \times 50 \mathrm{~m}^{2}$, which is then divided into 5 subplots (Figure 2). For vertical category $(\mathrm{dbh}>10 \mathrm{~cm})$, a subplot with measurement of $10 \times 10 \mathrm{~m}^{2}$ is used, but on the boundary $(\mathrm{dbh}<10)$ and low flora, a subplot with measurement of $5 \times 5$ $\mathrm{m}^{2}$ is used. The species and the quantity of plants in every sub plot are recorded. The diameter of high pectoral timbers is measured (dbh) and recorded. Measurement at the area covers the species, the quantity of individual on every species, the diameter of tree, and height of tree.

The measurement analysis of diversity of plants and low plants uses index as follow (Ismaini 2015), namely, the index of diversity of Shannon-Wiener (Magurran 1988):

$$
H^{\prime}=-\sum_{i=1}^{S}(p i)(\ln p i)
$$

Where, H': the result of diversity index of ShannonWiener and pi: proportion of every species i. so $\mathrm{H}^{\prime}$ is sum of whole pi ln pi for all species in community.

The Index of Species Evenness: $(E)=H^{\prime} / \ln S$, where E: index of species evenness, $\mathrm{H}^{\prime}$ : the result of diversity index of Shannon-Wiener, and S: sum of species which have been observed.

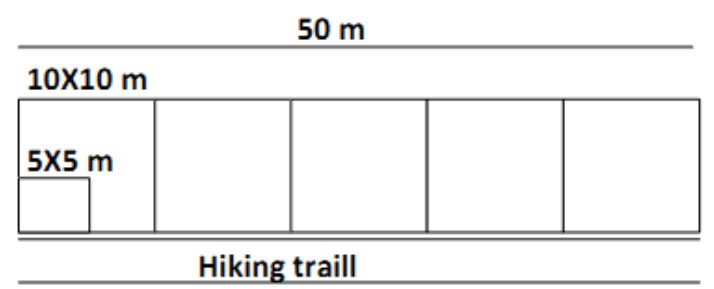

Figure 2. Plot sampling layout. Plot size: $10 X 50 \mathrm{~m}$.

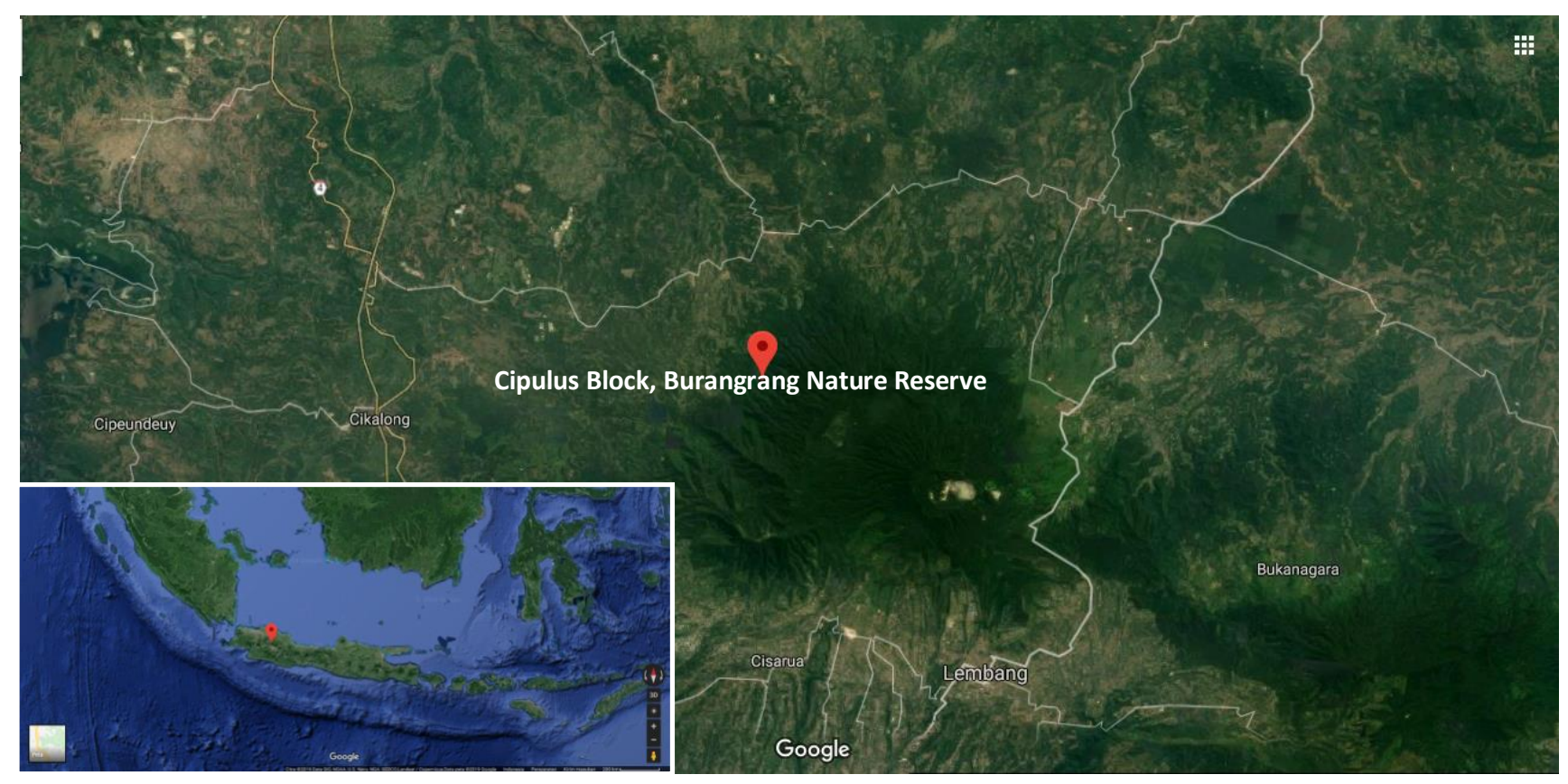

Figure 1. Location of Mt. Burangrang Nature Reserve, Purwakarta, West Java, Indonesia 


\section{Data analysis}

Analysis of structure and vegetation was done by counting Importance Value Index (IVI) by formula:

$$
\mathrm{IV}=\mathrm{RD}+\mathrm{RF}+\mathrm{RDo} \text {, }
$$

Where, RD: Relative Density, RF: Relative Frequency, RDo: Relative Dominance (\%). The Calculation of INP, according to Soerianegara and Indrawan (1982), is as follow:

$$
\begin{aligned}
& \mathrm{RD}=\frac{\text { number of individuals of a spesies }}{\text { total number of individual }} \times 100 \% \\
& \mathrm{RF}=\frac{\text { frequency of a species }}{\text { sum frequency of all species }} \times 100 \% \\
& \mathrm{RDo}=\frac{\text { dominance of a species }}{\text { dominance of all species }} \times 100 \%
\end{aligned}
$$

\section{RESULTS AND DISCUSSION}

\section{List of vascular plants in Mt. Burangrang Nature Reserve}

A total of 208 species of vascular plant (more or less $35 \%$ species of the western mountain flora of Java) belonging to 85 families have been recorded along transect on Block Cipulus Mt. Burangrang, both in the plots or outside the plots (Table 1). Based on the Angiosperm Phylogeny Group classification (1998; 2003; 2009; 2016), these species can be classified into: basal family of angiosperm (1 species), magnoliids (21 species), monocots (33 species), eudicots (1 species), superrosids (1 species), rosids (74 species), superasterids (5 species), and Asterids (47 species), plus 23 species of fern and 2 species of lycophytes. The gymnosperms group was not found in this observation.

Based on their habits, the tree type with 72 species has more member group than other types, such as: herbs, shrubs, climbing, epiphytes, creeping and palms type, with 56 species, 30 species, 27 species, 16 species, 5 species, and 2 species respectively. Lauraceae (10 species) and Fagaceae (2 species), the typical families in the submontane to montane forest, can also be found in this area. Other families with the high number of species members in study are Urticaceae (9 species), Rubiaceae (8 species), Moraceae (7 species), Orchidaceae (6 species), Phyllanthaceae (6 species), Arecaceae (6 species) and Araceae (6 species).

A total of 14 species belongs to IUCN redlist, with categories of least concern (12 species), vulnerable (1 species), and endangered (1 species). Furthermore, Castanopsis argentea, Pinanga javana and Amorphophallus decus-silvae are included in protected plants (Permen LHK No 20 tahun 2018). Furthermore, A. decus-silvae, wellknown as endemic flora of Java (Yuzzami et al. 2017), were found at flowering phase (up to $2.15 \mathrm{~m}$ high). Other species, i.e. Kadsura scanden and Calamus ciliaris belonging to 200 of rare species in Indonesia, were also found here (Mogea et al. 2011). Some of pterydophytes belong to IUCN red list based on an assesment by Fernando et al. (2008) in Philiphines, i.e. Asplenium nidus L. (VU), Cyathea contaminans (Wall. Ex Hook) Copel. (VU), Huperzia squarrosa (G. Forst.) Trevis. (EN), Aglaomorpha heraclea (Kuntze) Kopel. (VU), and Microsorum membranifolium (R.Br.) Ching (LC).

In addition to native species of Java, exotic species were also found in Mt. Burangrang Nature Reserve, such as Coffea arabica, C. canephora, Melastoma affine, Clidema hirta, Chromolaena odorata, Austroeupatorium inulifolium, Ageratina riparia, Lantana camara and Brugmansia suaveolens. Brugmansia suaveolens, $M$. acuminata, A. riparia, L. camara and C. odorata have been naturalized in Java for long years ago (Backer and Bakhuizen v.d. Brink 1963; 1965; 1968; Tjitrosoedirdjo et al. 2016). Firstly, these species are imported for medicinal, food or ornamental plants in the Dutch colonial through botanic gardens. Brugmansia suaveolens, for example, was imported from the Americas for ornamental plant (Bruggeman 1927; Dakkus 1930), but today, it grows wild on the banks of rivers, on moist areas or on little shade and it can also be found in various regions in Indonesia (Wahyuni and Tjitrosoedirdjo 2013; Zuhri and Mutaqien 2013; Junaedi 2014; Sutomo et al. 2018). Exotic species can threaten as invasive as reported in various regions in Indonesia (Setiawan and Sulistyawati 2008; Wahyuni and Tjitrosoedirdjo 2013; Zuhri and Mutaqien 2013; Tjitrosoedirdjo et al. 2016; Sutomo et al. 2018), so we need to be aware about this.

\section{Floristic composition and structure vegetation}

Diversity of tree in the observation plot in Mt. Burangrang Nature Reserve

A total of 48 individuals of tree (belong to 14 species from 10 family) were found in observation plot which were dominated by pioneer species i.e. Villebrunea integrifolia, Antidesma tetrandum, Acronychia trifoliolata and Dendrocnide stimulans (Table 2). It indicated that forest of lower submontane of Blok Cipulus Mt. Burangrang is disturbed or experienced secondary succession. Data of diameter distribution of tree (Figure 3) supported this statement too. Small trees $(\mathrm{dbh}<50 \mathrm{~cm})$ dominate more than large trees $(\mathrm{dbh}>50 \mathrm{~cm})$. The Shannon-Wienner's diversity index $\left(H^{\prime}\right.$ value $\left.=1.85\right)$ on tree level shows the result of medium category. While, the equality value only $0.35(\mathrm{~J}<0.5)$ indicates the spread of species that are not evenly distributed at the tree level.

Villebrunea integrifolia (Gaudich.) Miq. shows increasing trends on the number of individual in observed plot. Increases of individual can be seen from each plot and Villebrunea integrifolia (Gaudich.) Miq. has the highest number of individual which was found throughout the area. Furthermore, Plot 2 is the most distributed area containing Villebrunea integrifolia (Gaudich.) Miq., Antidesma tetrandum Bl., Acronychia trifoliolata Zoll \& Moritzi, Dendrocnide stimulans (L.f) Chew, and Litsea angulata Bl. Some species of lower submontane, such as, Litsea 
diversifolia Bl., L. mappacea Boerl. and L. angulata Bl. were also found here (Backer and Bakhuizen v.d. Brink 1963; Sunarto et al. 2019). For, the type of mountain flora, only 1 individual for each type were found, while Piper aduncum L., a well-known exotic species (Hartemink 2010), may only be found in one plot, but not in other plot. The distribution of $P$. aduncum species is more commonly found in riparian areas with a little light.

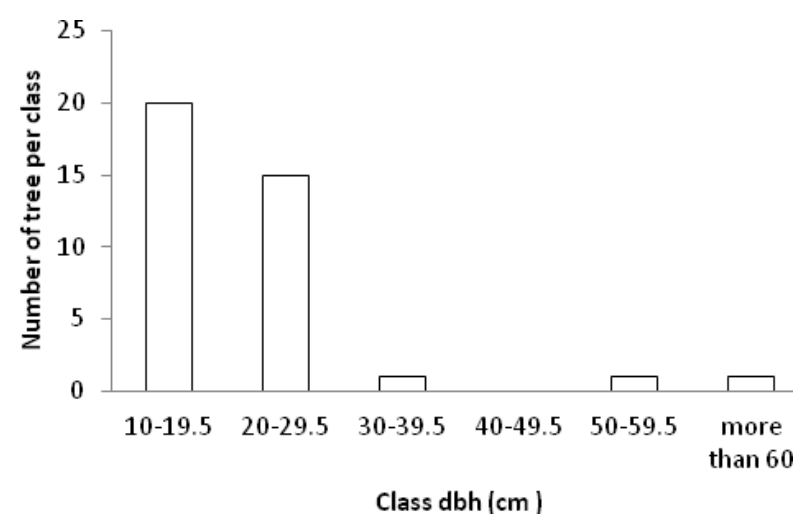

Figure 3. Frequency histogram of diameter distribution of tree (tree with dbh>10 cm)

Table 1. A list of vascular plant in Mt. Burangrang Nature Reserve, West Java, Indonesia both in the plots and outside observation plots

\begin{tabular}{|c|c|c|c|}
\hline Names of taxa & Habits & $\begin{array}{c}\text { Conserv. } \\
\text { status }\end{array}$ & $\begin{array}{l}\text { Coelogyne cf speciosa (Blume.) Lindl. } \\
\text { Corymborkis sp. } \\
\text { Eria sp. }\end{array}$ \\
\hline Basal Angiospermae & & & Habenaria angustifolia Kunth \\
\hline Schisandraceae Blume. & & & Liparis sp. \\
\hline Kadsura scandens (Blume.) Blume. & $\mathrm{Cl}$. & NE & Pandanaceae R.Br. \\
\hline Magnoliids & & & Freycinetia insignis Blume. \\
\hline Annonaceae Juss. & & & Freycinetia javanica Blume. \\
\hline Fissistigma latifolium (Dunal.) Merr. & $\mathrm{Cl}$. & $\mathrm{NE}$ & Poaceae Barnhart \\
\hline Polyalthia subcordata (Blume). Blume & Sh. & $\mathrm{NE}$ & Dinochloa scandens (Blume ex Nees) Kuntze \\
\hline Uvaria schizocalyx Back. & Sh. & $\mathrm{NE}$ & Isachne pangerangensis Zoll. \& Moritzi. \\
\hline Aristolochiaceae Juss. & & & Smilacaceae Vent. \\
\hline $\begin{array}{l}\text { Aristolochia coadunata Back. } \\
\text { Lauraceae Juss. }\end{array}$ & Cl. & NE & $\begin{array}{l}\text { Smilax leucophylla Blume. } \\
\text { Smilax zeylanica L. }\end{array}$ \\
\hline Cinnamomum porrectum (Roxb) Kosterm. & $\mathrm{T}$ & NE & Zingiberaceae Martinov. \\
\hline Cryptocarya ferrea Blume. & $\mathrm{T}$ & NE & Etlingera coccinea (B1.) S.Sakai \& Nagam \\
\hline Endiandra rubescens (Blume) Miq. & $\mathrm{T}$ & & Zingiber inflexum Blume. \\
\hline Litsea diversifolia Blume. & $\mathrm{T}$ & $\mathrm{NE}$ & Eudicots \\
\hline Litsea cf garciae Vidal. & $\mathrm{T}$ & $\mathrm{NE}$ & Proteaceae Juss. \\
\hline Litsea mappacea Boerl. & $\mathrm{T}$ & $\mathrm{NE}$ & Helicia robusta (Roxb) R.Br. ex Blume. \\
\hline Litsea noronhae Blume. & $\mathrm{T}$ & $\mathrm{NE}$ & Superrosids \\
\hline Neolitsea javanica Back. & $\mathrm{T}$ & $\mathrm{NE}$ & Altingiaceae \\
\hline Phoebe grandis (Nees) Merr. & $\mathrm{T}$ & $\mathrm{NE}$ & Altingia excelsa Noronha. \\
\hline $\begin{array}{l}\text { Tetranthera angulata (Blume) Ness. } \\
\text { Magnoliaceae Juss. }\end{array}$ & $\mathrm{T}$ & $\mathrm{NE}$ & $\begin{array}{l}\text { Rosids } \\
\text { Anacardiaceae R.Br. }\end{array}$ \\
\hline Magnolia sumatrana (Miq.) Figlar \& Noot. & $\mathrm{T}$ & $\mathrm{LC}$ & Semecarpus cuneiformis Blanco. \\
\hline Magnolia lillifera Druce. & $\mathrm{T}$ & $\mathrm{LC}$ & Begoniaceae C.Agardh \\
\hline Monimiaceae Juss. & & & Begonia isoptera Dryand \& Sm. \\
\hline $\begin{array}{l}\text { Kibara coreacea Hook.f \& Thomson. } \\
\text { Myristicaceae }\end{array}$ & $\mathrm{T}$ & $\mathrm{NE}$ & $\begin{array}{l}\text { Begonia longifolia Blume. } \\
\text { Begonia multangula Blume. }\end{array}$ \\
\hline Knema cinerea Warb. & $\mathrm{T}$ & $\mathrm{NE}$ & Brassicaceae Burnett \\
\hline Piperaceae Giseke & & & Cardamine hirsuta $\mathrm{L}$. \\
\hline Peperomia laevifolia (Bl) Miq. & Ep. & $\mathrm{NE}$ & Cannabaceae Martinov. \\
\hline Piper aduncum L. & $\mathrm{T}$ & $\mathrm{NE}$ & Trema orientalis (L.) Blume. \\
\hline
\end{tabular}

Piper sulcatum Blume.

Monocots

Amaryllidaceae J. St. Hill.

Curculigo capitulata (Lour.) Kuntze. $\quad$ H $\quad$ NE

Araceae Juss

Alocasia longiloba Miq.

Amorphophallus decus-silvae Backer \& Aldrew.

Homalomena pendula (Blume) Bahk.f.

Pothos scandes L.

Raphidophora $\mathrm{sp}$

Schismatoglottis acuminatissima Schott.

Arecaceae Berch. \& Presl.

Calamus ciliaris Blume.

Calamus heteroideus Blume.

Caryota mitis Lour.

Pinanga javana $\mathrm{Bl}$.

Plectocomia elongata Mart ex B1.

Asparagaceae Juss.

Cordyline fruticosa (L.) A. Chev.

Ophiopogon caulescens (B1.) Backer

Commelinaceae Mirb

Commelina palludosa $\mathrm{Bl}$.

Forrestia mollissima (B1.) Kord.

Costaceae Nakai

Costus speciosa (J.Konig) Sm.

Dioscoreaceae R.Br.

Tacca chantrieri Andre.

Musaceae Juss.

Musa acuminata Colla.

NE

$\mathrm{H}$ Protected

plant

$\mathrm{H} \quad \mathrm{NE}$

$\mathrm{H} \quad \mathrm{NE}$

$\mathrm{H} \quad \mathrm{NE}$

$\mathrm{H} \quad \mathrm{NE}$

Cl. NE

Cl. NE

Pl. LC

Pl. Protected

plant

Cl. NE

Cl. NE

$\mathrm{H} \quad \mathrm{NE}$

$\mathrm{H} \quad \mathrm{NE}$

$\mathrm{H} \quad \mathrm{NE}$

$\mathrm{H} \quad \mathrm{NE}$

$\mathrm{H} \quad \mathrm{NE}$

$\mathrm{H} \quad \mathrm{LC}$

Ep. NE

Ep. NE

$\mathrm{H} \quad \mathrm{NE}$

$\mathrm{H} \quad \mathrm{NE}$

$\mathrm{H} \quad \mathrm{NE}$

Ep. NE

Cl. NE

Cl. NE

Cl. NE

$\mathrm{H} \quad \mathrm{NE}$

Cl. NE

Cl. NE

$\mathrm{H} \quad \mathrm{NE}$

$\mathrm{H} \quad \mathrm{NE}$

Sh. NE

$\mathrm{T}$

NE

$\mathrm{T}$

NE

$\mathrm{H} \quad \mathrm{NE}$

$\mathrm{H} \quad \mathrm{NE}$

$\mathrm{H} \quad \mathrm{NE}$

Cr. NE

T LC 
Celastraceae R.Br.

Euonymus indicus B.Heyne ex Wall.

Clusiaceae Lindl.

Garcinia sp.

Cucurbitaceae Juss.

Gynostemma pentaphyllum (Thunb.) Makino

Melothria leucocarpa Blume.

Zehneria japonica (Thunb) H. Y.Liu

Cunoniaceae R.Br.

Weinmannia blumei Prantl.

Elaeocarpaceae Juss.

Elaeocarpus angustifolius Blume.

Elaeocarpus pirrei Kord \& Valeton

Euphorbiaceae Juss

Claoxylon longifolium (Blume) Endl ex Hassk.

Homalanthus populneus (Geiseler) Pax.

Macaranga tanarius Mull. Arg.

Ostodes paniculata $\mathrm{Bl}$.

Homalanthus giganteus Zoll \& Moritzi.

Fabaceae Lindl

Archidendron clypearia Jack \& Nielsen.

Derris elliptica (Wall) Benth.

Hylodesmum repandum (Vahl) H.Ohashi \&

R.R.Mill.

Paraserianthes lophantha (Wild) I.C.Nielsen.

Fagaceae Dumort.

Castanopsis argentea (Blume.) A.DC.

Lithocarpus elegans (Blume) Hatus ex Soepadmo

Juglandaceae DC ex. Perleb.

Engelhardtia spicata Lechen ex Blume

Malvaceae Juss.

Sida acuta Burm.f.

Sterculia coccinea Jack.

Sterculia foetida $\mathrm{L}$.

Sterculia rubiginosa Vent.

Melastomataceae Juss.

Clidemia hirta (L) D.Don.

Creochiton bibracteata (Blume.) Blume.

Melastoma affine D.Don.

Meliaceae Juss.

Aglaia eximia Miq.

Dysoxylum excelsum Blume.

Dysoxyllum nutans (B1.) Miq.

Moraceae Gaudich.

Ficus ampelas Burm.f.

Ficus heterophylla L.f.

Ficus laevicarpa Elme.

Ficus sp.1 (small fruit)

Ficus sp.2 (big fruit)

Ficus variegata Blume.

Maclura cochinchinensis (Lour). Corner.

Myrtaceae Juss.

Syzygium accuminatissimum (Blume.) DC.

Syzygium racemosum (Blume.)DC.

Syzygium rostratum (Blume.) DC.

Phyllanthaceae Martinov.

Antidesma tetrandum Blume.

Antidesma tomentosa Blume.

Bischofia javanica Blume.

Breynia microphylla (Kurzweil ex Teijsm. \&

Binn) Mull. Arg.

Bridelia insulana Hance.

Glochidion rubrum Blume.

Polygalaceae Hoffmanns \& Link

Polygala venenosa Juss ex Poir.

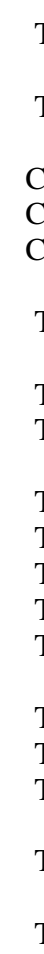

$\mathrm{T}$

T NE

T NE

Cl. NE

Cl. NE

Cl. NE

T NE

T NE

T NE

T NE

T NE

$\mathrm{T} \quad \mathrm{NE}$

T NE

$\mathrm{T} \quad \mathrm{NE}$

$\mathrm{T} \quad \mathrm{NE}$

T NE

T NE

EN,

protected

plant

NE

LC

$\mathrm{H}$

$\mathrm{T}$

$\mathrm{T}$

$\mathrm{T}$

H

Sh.

$\mathrm{T}$

$\mathrm{T}$

$\mathrm{T}$

Sh.

Sh.

Sh.

$\mathrm{T}$

$\mathrm{T}$

$\mathrm{T}$

$\mathrm{T}$

$\mathrm{T}$

T

Sh.

NE

$\mathrm{NE}$

$\mathrm{NE}$

$\mathrm{NE}$

NE

NE

NE

NE

NE

NE

NE

NE

NE

NE

NE

NE

NE

NE

$\mathrm{NE}$

$\mathrm{NE}$

NE

$\mathrm{NE}$

$\mathrm{NE}$

$\mathrm{NE}$

NE

Sh. NE
Rosaceae Juss.

Rubus fraxinifolius Hayata Cl. NE

Rubus rosiifolius $\mathrm{Sm}$.

Rutaceae Juss.

Acronychia trifoliolata Zoll \& Moritzi $\quad$ T $\quad$ LC

Luvunga sarmentosa Kurz. $\quad \mathrm{Cl}$. NE

Toddalia asiatica (L) Lamk. $\quad$ Cl. NE

Salicaceae Mirb.

Flacourtia rukam Zoll. \& Moritzi $\quad$ T $\quad$ NE

Sapindaceae Juss.

Acer laurinum Hassk.

Staphylleaceae Martinov.

Turpinia sphaerocarpa Hassk. $\quad$ T NE

Thymelaeaceae Juss.

Daphne composita (L.f.) Gilg.

Urticaceae Juss

Boehmeria nivea (L.) Gaudich.

Debregeasia longifolia (Burm.f) Wedd.

Dendrocnide stimulans (L.f) Chew.

Elatostema paludosum Miq.

Elatostema strigosum Hassk.

Oreocnide integrifolia (Gaudich) Miq.

Pilea melastomoides (Poir.). Wedd.

Poikilospermum suaveolens (Blume) Merr.

Villebrunea integrifolia Gaudich.

Vitaceae Juss

Leea angulata Korth ex Miq.

Leea indica (Burm.f) Merr.

Cissus bicolor Domin

Superasterids

Amaranthaceae Juss.

Achyranthes bidentata Blume. $\quad \mathrm{H} \quad \mathrm{NE}$

Iresine herbstii Hook.

Balanophoraceae A. Rich.

Balanophora elongata Blume.

Polygonaceae Juss.

Persicaria chinense (L.) H.Gross.

Persicaria nepalensis (Meisn). Miyabe

Asterids

Acanthaceae Juss

Peristrope hyssopifolia (Burm.f) Bremek

Strobilanthes paniculata Miq.

Actinidiaceae Gilg \& Werderm

Saurauia cauliflora DC.

Saurauia reinwardtiana Blume.

Adoxaceae

Sambucus javanica Blume.

Viburnum sambucinum Blume.

Apiaceae Lindl.

Hydrocotyle javanica Thunb.

Alyxia reindwarthii Blume.

Hoya multiflora Blume.

Parameria laevigata (Juss.). Moldenke.

Rauvolfia javanica Kord \& Valeton.

Thylophora villosa Blume.

Araliaceae Juss.

Arthrophyllum diversifolium Blume.

Schefflera rugosa (Blume) Harm.

Trevesia sundaica Miq.

Asteraceae Bercht. \& J.Presl

Ageratina riparia (Regel) R.M.King \& H.Rob. H HE

Chromolaena odorata (L) R.M.King \& H.Rob H NE

Vernonia arborea Buch-Ham. Sh. NE

Balsaminaceae A.Rich

Impatiens platypetala Lindl. $\quad \mathrm{H} \quad \mathrm{NE}$

Campanulaceae Juss.

Lobelia angulata Forst.
$\mathrm{H} \quad \mathrm{NE}$

$\mathrm{H} \quad \mathrm{NE}$

$\mathrm{H} \quad \mathrm{NE}$

$\mathrm{H} \quad \mathrm{NE}$

$\mathrm{H} \quad \mathrm{NE}$

T VU

T NE

Sh. NE

Cr. LC

Cl. NE

$\mathrm{Cl} \quad \mathrm{NE}$

$\mathrm{T} \quad \mathrm{NE}$

Cl. NE

T NE

Sh. NE

Sh. NE

Cr. NE 
Cornaceae Bercht. \& J.Presl

Alangium rotundifolium (Hassk.) Bloemb.

Gesneriaceae Rich. \& Juss.,

Aechynanthus cf radicans Jack.

Agalmyla parasitica (Lamk.) Kuntze

Cyrtandra pendula Blume.

Cyrtandra picta Blume

Rhynchoglossum obliquum Blume.

Lamiaceaea Martinov.

Clerodendrum inerme (L.) Gaertn.

Paraplomis oblongifolia (Blume) Prant.

Plantaginaceae Juss

Plantago major L.

Primulaceae Batsch ex Borkh

Embelia pergamacea A.DC.

Maesa latifolia A.DC.

Rubiaceae Juss

Coffea arabica $\mathrm{L}$.

Coffea canephora Pierre ex A. Froenhener

Ixora coccinea $\mathrm{L}$.

Lasianthus cf stipularis Blume.

Lasianthus stercorarius Blume.

Mussaenda frondosa $\mathrm{L}$.

Mycetia cauliflora Reinw

Rubia cordifolia $\mathrm{L}$.

Scrophulariaceae Juss.

Torenia asiatica L.

Stemonuraceae Karehed.

Gomphandra javanica (Blume.) Valeton

Solanaceae Juss.

Brugmansia suaveolens (Humb. \& Bonpl. ex Willd.) Bercht. \& J.Presl

Theaceae Mirb.

Camellia lanceolata (Blume.) Seem.

Gordonia excelsa (Blume.) Blume.

Pyrenaria serrata Blume

Schima wallichi (DC) Kort.

Verbenaceae J.St.Hil.

Lantana camara $\mathrm{L}$.

Fern

Aspleniaceae Newman

Asplenium tenerum (G. Forst.) var. pallidum

(Blume.) Veldk. \& Wardani
Asplenium pellucidum Lamk.

Asplenium nidus L.

Asplenium salignum Blume.

Hymenasplenium unilterale Lamk.

Athyriaceae Alston

Diplazium esculentum (Retz.) Sw.

Diplazium dilatatum Blume.

Diplazium sp.

Cyatheaceae Kaulf.

Cyathea contaminans (Wall. Ex Hook) Copel.

Davalliaceae M.R.Schomb.

Davallia denticulata (Burm.f.) Mett. ex Kuhn. Ep. NE

Dennstaedtiaceae Lotsy

Dennstaedtia scandens (B1.) T. Moore $\quad$ Cr. $\quad$ NE

Dryopteridaceae Herter

Bolbitis sp.

Dryopteris sp.

Hymenophyllaceae Mart.

$\begin{array}{ll}\text { Sh. } & \text { NE } \\ \text { Sh. } & \text { LC }\end{array}$

Sh. NE

Sh. NE

Sh. NE

Sh. NE

Sh. NE

Sh. NE

$\mathrm{H} \quad \mathrm{NE}$

T NE

$\mathrm{H} \quad \mathrm{NE}$

Cephalomanes javanicum C. Presl.

Marattiaceae Kaulf.

Angiopteris avecta (G. Forst.) Hoffm.

Nephrolepidaceae Pic.Serm.

Nephrolepis bisserata (Sw.) Scott.

Polypodiaceae Berecht. \& J. Presl.

Pyrrosia albicans (Blume) Ching

Goniophlebium persicifolium (Desv.) Bedd.

Aglaomorpha heraclea (Kuntze) Kopel.

Microsorum membranifolium (R.Br.) Ching

Pteridaceae E.D.M.Kirchn.

Antrophyum reticulatum (Forst.) Kaulf.

Pteris longipinnula Wall. ex J. Agardh

Thelypteridaceae Ching ex Pic. Serm.

Chingia ferox (B1.) Holttum

Ep. NE

Ep. $\quad \mathrm{VU}^{*}$

Ep. NE

$\mathrm{H} \quad \mathrm{NE}$

$\mathrm{H} \quad \mathrm{NE}$

$\mathrm{H} \quad \mathrm{NE}$

$\mathrm{H} \quad \mathrm{NE}$

$\mathrm{T} \quad \mathrm{VU}^{*}$

Lycophytes

$\begin{array}{cc}\text { Sh. } & \text { NE } \\ \text { T } & \text { NE } \\ \text { T } & \text { LC } \\ \text { T } & \text { LC }\end{array}$

Lycopodiaceae P.Beauv. ex Mirb.

Huperzia squarrosa (G. Forst.) Trevis.

Selaginellaceae Willk.

Selaginella sp.

$\mathrm{H} \quad \mathrm{NE}$

$\mathrm{H} \quad \mathrm{NE}$

$\mathrm{H} \quad \mathrm{NE}$

Sh. NE

Ep. NE

Ep. NE

Ep. NE

Ep. $\quad \mathrm{VU}^{*}$

Ep. LR*

Ep. NE

$\mathrm{H} \quad \mathrm{NE}$

$\mathrm{H} \quad \mathrm{NE}$

Note: T: Tree, Sh: Shrub, H: Herbs, Ep: Epiphytes, Pl: Palm, Cl:

Climbing, Cr: Creeping, LC: Least concerns, VU:Vunereable,

EN: Endangered, *according to Fernando et al. (2008)

Table 2. Species distribution of tree in Plot 1, Plot 2, Plot 3 and Plot 4 in Mt. Burangrang Nature Reserve, West Java, Indonesia

\begin{tabular}{|c|c|c|c|c|c|c|c|c|}
\hline \multirow{2}{*}{ Scientific names } & \multirow{2}{*}{ Family } & \multicolumn{4}{|c|}{ Number of individual } & \multirow{2}{*}{ IV } & \multirow{2}{*}{$\begin{array}{c}\text { Basal } \\
\text { area } \\
\left(\mathbf{m}^{2}\right)\end{array}$} & \multirow{2}{*}{$\begin{array}{l}\text { Mean } \\
\text { hight } \\
\text { (m) }\end{array}$} \\
\hline & & Plot 1 & Plot 2 & Plot 3 & Plot 4 & & & \\
\hline Villebrunea integrifolia (Gaudich.) Miq. & Urticaceae & 1 & 5 & 7 & 11 & 121.05 & 388.63 & 9.17 \\
\hline Antidesma tetrandum $\mathrm{Bl}$. & Phyllanthaceae & - & 6 & - & - & 30.26 & 35.91 & 11.83 \\
\hline Acronychia trifoliolata Zoll \& Moritzi & Rutaceae & 1 & 3 & - & - & 27.19 & 11.65 & 12.83 \\
\hline Dendrocnide stimulans (L.f) Chew & Urticaceae & - & 2 & 1 & - & 23.03 & 5.86 & 6.83 \\
\hline Polyalthia rumphii (Bl. ex Hensch) Merr. & Annonaceae & - & - & - & 1 & 13.60 & 1.13 & 10.30 \\
\hline Ficus sp. & Moraceae & 1 & - & - & - & 9.43 & 259.40 & 25.00 \\
\hline Cyathea contminans (Wall. Ex Hook.) Copel. & Cyatheaceae & - & - & 2 & - & 9.43 & 7.17 & 5.85 \\
\hline Litsea diversifolia $\mathrm{Bl}$. & Lauraceae & - & - & 1 & - & 9.43 & 4.91 & 10.50 \\
\hline Ficus variegata $\mathrm{Bl}$. & Moraceae & - & - & - & 1 & 9.43 & 2.55 & 7.20 \\
\hline Alangium rotundifolium (Hassk.) Bloemb. & Alangiaceae & 1 & - & - & - & 9.43 & 1.43 & 15.20 \\
\hline Litsea mappacea Boerl. & Lauraceae & 1 & - & - & - & 9.43 & 1.33 & 9.20 \\
\hline Litsea angulata $\mathrm{Bl}$. & Lauraceae & - & 1 & - & - & 9.43 & 0.95 & 7.50 \\
\hline Dysoxylum excelsum $\mathrm{Bl}$. & Meliaceae & - & - & 1 & - & 9.43 & 0.79 & 13.30 \\
\hline Piper aduncum L. & Piperaceae & 1 & - & - & & 9.43 & 0.79 & 12.05 \\
\hline Total & & 6 & 17 & 12 & 14 & & 722.50 & \\
\hline
\end{tabular}

Notes: IV: importance value 
The density level and number of tree species in Mt. Burangrang show lower value than other forest type in the similar altitude in Java (Tabel 3). Likewise, the canopy covering in the tree level shows a "gap" in almost all observation plots. It brings to a conclusion that vegetation in Mt. Burangrang has been disrupted and has come to a succession period as it has happened in Mt. Wilis (Purwaningsih et al. 2017). The smaller plot area causing low density and species were found.
Domination of mountain flora, ex. Aglaia eximia and Polyalthia subcordates, showed a positive regeneration trend compared with a high trees (Table 4). Polyalthia subcordata, Sambucus javanica, and Aglaia eximia were commonly found on those heights, as reported by Zuhri et al. (2018) in the remnant forest of Cibodas Botanic Gardens. Pinanga javana (Pinang jawa), is also spread throughout observation plots. Pinanga javana grows from low land to high land on Mt. Slamet area, Baturaden (Zulkarnaen et al. 2019).

Table 3. The comparison of density and number of tree species in submontane forest Mt. Burangrang Nature Reserve and other submontane forests in Java, Indonesia

\begin{tabular}{lccccccc}
\hline Parameters & $\begin{array}{c}\text { Present study } \\
\text { Mt. Burangrang }\end{array}$ & $\begin{array}{c}\text { Purwaningsih } \\
\text { et al. (2017) } \\
\text { Mt. Wilis }\end{array}$ & $\begin{array}{c}\text { Helmi et al. } \\
(\mathbf{2 0 0 9 )} \\
\text { Bodogol }\end{array}$ & $\begin{array}{c}\text { Suryanti } \\
\mathbf{( 2 0 0 6 )} \\
\text { Mt. Kendeng }\end{array}$ & $\begin{array}{c}\text { Suryanti } \\
(\mathbf{2 0 0 6})\end{array}$ & $\begin{array}{c}\text { Suryanti } \\
\mathbf{( 2 0 0 6 )} \\
\text { Malang }\end{array}$ & $\begin{array}{c}\text { Rahayoe } \\
(\mathbf{1 9 9 6}) \\
\text { Mt. Panenjoan }\end{array}$ \\
\hline Citalahab \\
\hline Plot size (ha) & $946-1104$ & 1100 & 806 & 1000 & 1000 & 1000 & $1000-1.200$ \\
Density (tree/ha) & 0.2 & 0.25 & 1.0 & 1.0 & 1.0 & 1.0 & 0.7 \\
Number of species & 190 & 836 & 352 & 406 & 421 & 405 & 395 \\
\hline
\end{tabular}

Table 4. Species distribution of seedling of some trees in Plot 1, Plot 2, Plot 3 and Plot 4 in Mt. Burangrang Nature Reserve, West Java, Indonesia and its importance value

\begin{tabular}{|c|c|c|c|c|c|c|c|c|c|}
\hline \multirow{2}{*}{ Scientific names } & \multicolumn{4}{|c|}{ Number of individu } & \multirow{2}{*}{ NI } & \multirow{2}{*}{ RD } & \multirow{2}{*}{$\mathbf{R F}$} & \multirow{2}{*}{ RDo } & \multirow{2}{*}{ IV } \\
\hline & Plot 1 & Plot 2 & Plot 3 & Plot 4 & & & & & \\
\hline Aglaia eximia Miq. & 80 & - & 3 & 4 & 87 & 25.22 & 3.76 & 5.15 & 34.13 \\
\hline Polyalthia subcordata (Blume.) Blume. & 21 & 5 & 1 & 4 & 31 & 8.99 & 6.77 & 9.28 & 25.03 \\
\hline Ficus sp. & 37 & - & - & - & 42 & 12.17 & 3.76 & 5.15 & 21.09 \\
\hline Endiandra rubescens (Blume) Miq. & 7 & - & 9 & - & 16 & 4.64 & 3.76 & 5.15 & 13.55 \\
\hline Engelhardtia spicata Lechen ex Blume & 6 & - & 5 & - & 11 & 3.19 & 3.01 & 4.12 & 10.32 \\
\hline Leea indica (Burm.f.) Merr. & - & 12 & - & 6 & 11 & 3.19 & 3.01 & 4.12 & 10.32 \\
\hline Sambucus javanica Blume. & - & 1 & 3 & 6 & 10 & 2.90 & 3.01 & 4.12 & 10.03 \\
\hline Pinanga javana $\mathrm{Bl}$. & - & 4 & 3 & 1 & 8 & 2.32 & 3.01 & 4.12 & 9.45 \\
\hline Cyathea contaminans (Wall. Ex Hook) Copel. & - & 4 & 2 & - & 6 & 1.74 & 3.01 & 4.12 & 8.87 \\
\hline Dysoxyllum nutans (B1.) Miq. & 14 & - & - & - & 14 & 4.06 & 1.50 & 2.06 & 7.62 \\
\hline
\end{tabular}

Note: NI: Number of Individu, RD: Relative Density, RF: Relative frequency, RDo: Relative Dominance, IV: Importance value

Table 5. Ten species of undergrowth vegetation with the highest importance value

\begin{tabular}{|c|c|c|c|c|c|c|c|c|c|}
\hline \multirow{2}{*}{ Scienfitic names } & \multicolumn{4}{|c|}{ Number of individu } & \multirow[b]{2}{*}{ NI } & \multirow[b]{2}{*}{ RD } & \multirow[b]{2}{*}{$\mathbf{R F}$} & \multirow{2}{*}{ RDo } & \multirow[b]{2}{*}{ IV } \\
\hline & Plot 1 & Plot 2 & Plot 3 & Plot 4 & & & & & \\
\hline Achyranthes bidentata Blume. & - & 16 & 33 & 88 & 137 & 9.33 & 5.46 & 5.46 & 20.26 \\
\hline Elatostema strigosum Hassk. & 63 & 21 & - & 88 & 172 & 11.72 & 3.83 & 3.83 & 19.37 \\
\hline Homalomena pendula (Blume) Bahk.f. & 43 & 28 & 25 & 10 & 106 & 7.22 & 6.01 & 6.01 & 19.24 \\
\hline Etlingera coccinea (B1.) S.Sakai \& Nagam & 18 & 57 & 17 & - & 92 & 6.27 & 6.01 & 6.01 & 18.29 \\
\hline Boehmeria nivea (L.) Gaudich. & 6 & 38 & 25 & 23 & 92 & 6.27 & 5.46 & 5.46 & 17.20 \\
\hline Commelina paludosa Blume & - & 34 & 16 & 2 & 52 & 3.54 & 5.46 & 5.46 & 14.47 \\
\hline Schismatoglottis acuminatissima Schott. & - & 57 & 31 & 4 & 92 & 6.27 & 3.83 & 3.83 & 13.92 \\
\hline Elatostema paludosum Miq. & - & - & 37 & 92 & 129 & 8.79 & 2.19 & 2.19 & 13.16 \\
\hline Psychotria angulata Korth & - & 3 & 47 & 5 & 55 & 3.75 & 3.83 & 3.83 & 11.40 \\
\hline Coffea arabica $\mathrm{L}$. & 98 & - & - & - & 100 & 6.81 & 2.19 & 2.19 & 11.18 \\
\hline
\end{tabular}

Note: NI: Number of Individu, RD: Relative Density, RF: Relative frequency, RDo: Relative Dominance, IV: Importance value 


\section{Diversity of undergrowth in the observation plot in Mt. Burangrang Nature Reserve}

The existence of undergrowth species plays an important role in maintaining the microclimate in the land. Achyranthes bidentata Blume., Elatostema strigosum Hassk., Homalomena pendula (Blume) Bahk.f., Etlingera coccinea (Bl.) S. Sakai \& Nagam and Boehmeria nivea (L.) Gaudich are the 10th species that dominate the forest of Mt. Burangrang (Table 5). The high value of importance shows the success of a plant in controlling the area by growing and developing with the habitat which has high humidity and relatively acid-netral $\mathrm{pH}$, although there were some wider gaps in some areas due to fallen trees. This shows that Achyranthes bidentata Blume is a dominant crop, with wide adaptability and tolerance to environmental factors (Ismaini et al. 2015). But, there was also a threat from the plants having a high growth rate such as Etilingera coccinea which has the potential as an invasive species as reported in several regions in Indonesia and this needs attention. Etlingera coccinea has a tight rhizome and forms a population that can inhibit other types of growth (Zuhri and Mutaqien 2013; Tjitrosoedirdjo et al. 2016).

Homalomena pendula and Boehmeria nivea were found throughout the observation plot. The production of large amounts of seeds can accelerate the spread of these species even further into the forest. Coffee arabica was only be found in plot I, which was directly adjacent to community plantations and the plot was a former plantation land cultivated by communities that had been planted with coffee decades ago.

The conclusion, Mt. Burangrang Nature Reserve stores a large variety of vascular plants including some rare plants as listed in IUCN redlist and nationally, but the condition of the forest in this zone shows disturbed forest which needs recovery as soon as possible. The presence of invasive trees could be slow down the succession toward climax. Further, research on interaction of biodiversity and surrounding community need to be done later to support the continuation of forest life in this area.

\section{ACKNOWLEDGEMENTS}

The author thank the head of the biology department and Isma Dwi Kurniawan, who has provided funding for this research through student research assistance programs, Department of Biology, Science and Technology faculties. chairman Balai Besar Konservasi Sumber Daya Alam (Nature Conservation Agency; West Java) for giving research permission to Burangrang Nature Reserve. The author also thank Tatang and Oding (Mt. Burangrang NR), Muslim (Cibodas Botanic Gardens), who has helped material collection in the field.

\section{REFERENCES}

APG I. 1998. An ordinal classification for the families of flowering plants. Ann Missouri Bot Gard 85: 531-553.
APG II. 2003. An update of the Angiosperm Phylogeny Group classification for the orders and families of flowering plants: APG II. Bot J Linn Soc 141: 399-436.

APG III. 2009. An update of the Angiosperm Phylogeny Group classification for the orders and families of flowering plants. APG III. Bot J Linn Soc 161: 105-121.

APG IV. 2016. An update of the Angiosperm Phylogeny Group classification for the orders and families of flowering plants: APG IV. Bot J Linn Soc 181: 1-20.

Backer CA, Posthumus O. 1939. Varenflora voor Java.. Archipel Drukkerij, Buitenzorg, NL.

Backer CA, van den Brink Jr. RCB. 1963. Flora of Java (Spermatophytes only) vol I. NVP Noordhoff, Groningen, The Netherland.

Backer CA, van den Brink Jr. RCB. 1965. Flora of Java (Spermatophytes only) vol II. NVP Noordhoff, Groningen, The Netherland.

Backer CA, van den Brink Jr. RCB. 1968. Flora of Java (Spermatophytes only) vol III. NVP Noordhoff, Groningen, The Netherland.

BBKSDA. 2016. Informasi Kawasan Konservasi lingkup BBKSDA Jabar: Cagar Alam Gunung Burangrang. BBKSDA, Bandung.

Bruggeman MLA. 1927. 'S lands plantentuin Buitenzorg-Gids voor den Bergtuin te Tjibodas, Sindanglaja. Archipel Drukkerij, Buitenzorg.

Christenhusz MJM, Zhang XC, Schneider H. 2011. A linear sequence of extant families and genera of lycophytes and ferns. Phytotaxa 19: 754

Dakkus PMW. 1930. An Alphabetical List of Plants Cultivated in Botanic Gardens, Buitenzorg. Archipel Drukkerij, Buitenzorg.

Girmansyah D. 2008. Keanekaragaman jenis Begonia (Begoniaceae) liar di Jawa Barat. Berita Biologi 9 (2): 195-203. [Indonesian]

Flora Malesiana. 2012. Flora Malesiana series II-Fern and Fern Allies Vol. 4. Flora Malesiana Foundation, The Nederland.

de Vogel EF. 1987. Manual of Herbarium Taxonomy: Theory and practice. UNESCO Regional Office for Science and Technology for South East Asia, Jakarta.

Hartemink AE. 2010. The invasive shrub Piper aduncum in PaPua New Guinea: a review. J Trop For Sci 22 (2): 202-213.

Fernando ES., Co LL, Lagunzad DA, Gruezo WS, Barcelona JF, Madulid DA, Lapis AB, Texon GI, Manila AC, Zamora PM. 2008. Threatened plants of the Philippines: a preliminary assessment. Asia Life Sci Suppl 3: 1-52.

Helmi N, Kartawinata K, Samsoedin I. 2009. An undescribed lowland natural forest at Bodogol, Gunung Gede Pangrango National Park, Cibodas Biosphere Reserve, West Java, Indonesia. Reinwardtia 13 (1): 33-46.

Hadiah JT. 2007. Systematics of Elatostema (Urticaceae). [Thesis]. University of New South Wales, New South Wales.

Holttum RE. 1966. A Revised Flora of Malaya. Volume II. Government Printing Off, Singapore.

Ismaini L, Lailati M, Rustandi, Sunandar D. 2015. Composition and plant diversity on Mount Dempo, South Sumatra. Pros Sem Nas Masy Biodiv Indon 1: 1397-1402.

Junaedi DI. 2014. Exotic plants in the Cibodas Botanic Gardens remnant forest: Inventory and cluster analysis of several environmental factors. Buletin Kebun Raya 17 (1): 1-8.

Magurran AE. 1988. Ecological Diversity and its Measurement. Princeton University Press, New Jersey.

Mansur M, Hidayati N, Juhaeti T. 2011. Struktur dan komposisi vegetasi pohon serta estimasi biomassa, kandungan karbon dan laju fotosintesis Di Taman Nasional Gunung Halimun-Salak. J Tek Ling 12 (2): 161-169.

Mansur M, Kartawinata K. 2017. Phytosociology of a lower montane forest on Mt. Batulanteh, Sumbawa, Indonesia. Reinwardtia 16 (2): 77-92.

Mogea JP, Gandawidjaja D, Wiriadinata H, Naution RE, Irawati. 2001. Tumbuhan Langka Indonesia. Herbarium Bogoriense, Bogor.

Setiawan NN, Sulistyawati E. 2008. Succession following reforestation on abandoned fields in Mount Papandayan, West Java. Procceding of International Conference on Environmental Research and Technology (ICERT 2008). Penang, Malaysia.

Purwaningsih, Plosokan R, Yusuf Razali, Kartawinata K. 2017. Phytosociological study of the montane forest on the south slope of Mt. Wilis, East Java. Indonesia. Reinwardtia 16 (1): 31-45.

Rothfels CJ, Sundue MA, Kuo LY, Larsson A, Kato, Schuettpelz E, Pryer KM. 2012. A revised family-level classification for eupolypod II ferns (Polypodiidae: Polypodiales). Taxon 2012: 1-19.

Rugayah, Retnowati A, Windadri FI, Hidayat A. 2004. Pengumpulan Data Taksonomi. In: Rugayah, Widjaja EA, Praptiwi (eds.). Pedoman 
Pengumpulan Data Keanekaragaman Flora. Pusat Penelitian BiologiLIPI, Bogor. [Indonesian]

Soerianegara I, Indrawan. 1982. Ekologi hutan Indonesia. Departemen Manajemen Hutan, Fakultas Kehutanan Institut Pertanian Bogor, Bogor. [Indonesian]

Tjitrosoedirdjo SS, Mawardi I, Tjitrosoedirdjo. 2016. 75 Important Invasive Plant Species in Indonesia. SEAMEO BIOTROP, Bogor.

Tsujino R, Yumoto T, Kitamura S, Djamaluddin I, Darnaedi D. 2016. History of forest loss and degradation in Indonesia. Land Use Pol 57: 335-347.

van Welzen PC, Raes N. 2011. The floristic position of Java. Gardens' Bulletin Singapore 63 (1\&2): 329-339.

Wahyuni I, Tjitrosoedirdjo SS. 2013. Observation on the development of important weeds and invasive alien plant species in Indonesia. Procceding 24th Asian-Pacific Weed Science Society Conference, October 22-25, 2013. Bandung [Indonesia]. van Steenis CGGJ. 1972. The Mountain Flora of Java. Koninklijke Brill NV. Leiden, The Netherlands

van Steenis CGGJ. Mountain Flora of Java, 2nd ed. Brill, Leiden.

Zhu H, Roos MC, Ridsdale CE. 2012. A taxonomic revision of the Malesian species of Lasianthus (Rubiaceae). Blumea 57: 1-102.

Zuhri M, Mutaqien Z. 2011. Perubahan komposisi vegetasi dan struktur pohon pada Plot Meijer (1959-2009) di Gunung Gede, Jawa Barat. Buletin Kebun Raya 14 (1): 37-45. [Indonesian]

Zuhri M, Mutaqien Z. 2013. The spread of non-native plant species collection of Cibodas Botanical Garden into Mt. Gede Pangrango National Park. J Trop Life Sci 3 (2): 74-82.

Zuhri M, Mutaqien Z, Nurdiana DR, Destri, Nudin, Djuanda. 2018. Vegetasi tumbuhan pada kawasan tepi hutan Taman Nasional Gunung Gede Pangrango yang berbatasan dengan Kebun Raya Cibodas. Biogenesis 6 (2): 105-114. [Indonesian] 